\title{
Implications of the generation of internal gravity waves by penetrative con- vection for the internal rotation evolution of low-mass stars
}

\author{
Charly Pinçon ${ }^{1, \star}, K_{\text {Kévin Belkacem }}^{1}$, and Marie-Jo Goupil ${ }^{1}$ \\ ${ }^{1}$ LESIA, Observatoire de Paris, PSL Research University, CNRS, Université Pierre et Marie Curie, Université Paris Diderot, 92195 \\ Meudon, France
}

\begin{abstract}
Due to the space-borne missions CoRoT and Kepler, noteworthy breakthroughs have been made in our understanding of stellar evolution, and in particular about the angular momentum redistribution in stellar interiors. Indeed, the high-precision seismic data provide with the measurement of the mean core rotation rate for thousands of low-mass stars from the subgiant branch to the red giant branch. All these observations exhibit much lower core rotation rates than expected by current stellar evolution codes and they emphasize the need for an additional transport process. In this framework, internal gravity waves (herefater, IGW) could play a signifivative role since they are known to be able to transport angular momentum. In this work, we estimate the efficiency of the transport by the IGW that are generated by penetrative convection at the interface between the convective and the radiative regions. As a first step, this study is based on the comparison between the timescale for the waves to modify a given rotation profile and the contraction/expansion timescale throughout the radiative zone of $1.3 \mathrm{M}_{\odot}$ stellar models. We show that IGW, on their own, are inefficient to slow down the core rotation of stars on the red giant branch, where the radiative damping becomes strong enough and prevent the IGW from reaching the innermost layers. However, we find that IGW generated by penetrative convection could efficiently modify the core rotation of subgiant stars as soon as the amplitude of the radial differential rotation between the core and the base of the convective zone is high enough, with typical values close to the observed rotation rates in these stars. This result argues for the necessity to account for IGW generated by penetrative convection in stellar modeling and in the angular momentum redistribution issue.
\end{abstract}

\section{Introduction}

Asteroseismology is a powerful tool to probe the internal structure of stars and the seismic data provided by the space-borne missions CoRoT and Kepler turn out to be a goldmine of information for the stellar evolution theory. The detection of mixed modes, which have amplitude both in the innermost layers and in the envelopes of stars, made the measurement of the mean core rotation possible for thousands of stars from the subgiant branch to the red giant branch. The observations clearly show that the mean core rotation moderately increases on the subgiant branch [1, 2] before it strongly drops along the red giant branch [3]. These observational facts are still unexplained by theoretical predictions. Indeed, transport of angular momentum by meridional circulation and shear-induced mixing as included in the current stellar evolution codes are unable to counteract the strong acceleration due to the core contraction [e.g. 4, 5]. Therefore, another mechanism must be found to efficiently slow the core rotation of these stars.

In this framework, internal gravity waves (hereafter, IGW), which are buoyancy waves propagating throughout the radiative zone of stars, may have a significative role to play. Indeed, in presence of differential rotation, IGW are radiatively damped, deposit the angular momentum that they carry into the medium and so can locally modify the internal rotation rate. Two main excitation mechanisms are usually invoked for these waves. Firstly, IGW can be generated by turbulent pressure in the convective bulk [6]. Turbulence-induced IGW have already been shown to be able to explain the quasi-flat rotation profile observed in the solar radiative zone [7, 8]. However, previous work [9] showed that the radiative damping drastically increases near the H-burning shell as the star evolves and should result in a rapid decoupling between these waves and the stellar core as soon as the beginning of the subgiant branch. Secondly, IGW can also be generated by the penetration of convective plumes into the top of the radiative zone. While penetrating by inertia, the convective plumes are slowed down by buoyancy braking and can transfer their kinetic energy into waves, as observed in geophysics or in numerical simulations [e.g. 10-12]. A semianalytical estimate of the generation of IGW by penetrative convection has already demonstrated the ability of these waves to efficiently affect the rotation of the solar radiative zone [13]. Nevertheless, an extension to more evolved stars is still missing.

\footnotetext{
^e-mail: charly.pincon@obspm.fr
} 
Here, we investigate the ability of plume-induced waves to modify a given rotation profile in subgiant and red giant stars. As a first step in the investigation, our study is based on the comparison of the dynamical wavedriven timescale with the contraction/expansion timescale throughout $1.3 \mathrm{M}_{\odot}$ stellar models. Moreover, the role of the radial differential rotation amplitude on the transport by IGW is stressed out.

\section{Timescales and wave flux generated by convective plumes}

\subsection{Characteristic timescales}

The local contraction/expansion timescale is equal to the characteristic timescale of evolution of the internal rotation rate in the case of local conservation of angular momentum. It is given by

$$
t_{\text {cont }}(r)=\left|\frac{r}{2 \dot{r}}\right|
$$

with $r$ the radius and $\dot{r}$ the Lagrangian radial velocity of a mass shell at the radius $r$. The local timescale associated with the transport of angular momentum by IGW, given a rotation profile $\Omega(r)$ (within the shellular approximation), is equal to the ratio of the density of angular momentum in the star to the radial divergence of the angular momentum wave flux, i.e.

$$
t_{w}(r) \sim\left|\frac{\rho r^{2} \Omega}{\dot{J}}\right|
$$

with $\rho$ the density at the equilibrium and $\dot{J}$ the divergence of the mean radial wave flux of angular momentum whose computation requires the knowledge of the wave energy flux, given below by Eq. (4). Therefore, IGW can be said to locally counteract the action of the contraction or the dilatation in the star if $t_{w} \leq t_{\text {cont }}$. Thus, the comparison of both timescales gives a first hint about the efficiency of the transport of angular momentum by IGW throughout the radiative zone of low-mass stars.

\subsection{Wave flux of angular momentum}

Convective plumes are strong coherent downwards flows that originate from the uppermost layers of low-mass stars and develop across their convective envelope by turbulent entrainment of matter. Once they reach the border with the underlying radiative zone, they penetrate by inertia into the stably stratified layers where they are slowed down by buoyancy braking. This process converts a part of the plume kinetic energy into wave energy, which can then propagate into the radiative zone. To model this excitation mechanism, [13] considered the pressure exerted by an ensemble of incoherent convective plumes in the penetration zone as the source term in the wave equation. By assuming a high Péclet number and a very sharp thermal transition at the base of the convective zone, the mean radial wave energy flux per unit of frequency, for an angular degree $l$ and an azimuthal number $m$ at the top of the radiative zone, can be written as

$\mathcal{F}_{E, w}\left(r_{t}, \omega, l, m\right) \sim \frac{1}{4 \pi r_{t}^{2}} \frac{\mathcal{A} \mathcal{S}_{p}}{2} \frac{\rho_{b} V_{b}^{3}}{2} F_{R, l} \frac{e^{-\omega^{2} / 4 v_{p}^{2}}}{v_{p}} e^{-l(l+1) b^{2} / 2 r_{t}^{2}}$,

where $r_{t}$ is the radius at the top of the radiative zone, $\mathcal{A}$ is the plumes filling factor in the excitation region, $\mathcal{S}_{p}=\pi b^{2}$ is the horizonthal area occupied by one single plume, with $b$ the plume radius, $\rho_{b}$ and $V_{b}$ are respectively the density and the plume velocity at the base of the convective region, $F_{R, l}=\sqrt{l(l+1)} V_{b} / r_{t} N_{0}$, with $N_{0}$ the Brunt-Väisälä frequency at the top of the radiative zone, and $v_{p}=1 / \tau_{p}$, with $\tau_{p}$ the plume lifetime.

Each spectral component of the total wave energy flux emitted from the top of the radiative zone are radiatively damped as it propagates through the radiative region and so contributes to the transport of angular momentum in presence of differential rotation. Then, the total wave energy flux at a radius $r$ can be expressed following [7]

$$
\mathcal{F}_{J, w}(r)=\sum_{l} \sum_{m=-l}^{m=+l} \int_{-\infty}^{+\infty} \frac{m}{\omega} \frac{r_{t}^{2}}{r^{2}} \mathcal{F}_{E, w}\left(r_{t}, \omega, l, m\right) e^{-\tau(r, \hat{\omega}, l)} \mathrm{d} \omega,
$$

with

$$
\tau(r, \hat{\omega}, l)=[l(l+1)]^{3 / 2} \int_{r}^{r_{t}} K \frac{N N_{T}^{2}}{\hat{\omega}^{4}}\left(\frac{N^{2}}{N^{2}-\hat{\omega}^{2}}\right)^{1 / 2} \frac{\mathrm{d} r}{r^{3}},
$$

where $N$ is the Brunt-Väisälä frequency, with its thermal part $N_{T}, K$ is the radiative diffusion coefficient, and

$$
\hat{\omega}(r, \omega, m)=\omega-m \delta \Omega(r)
$$

is the Doppler-shifted intrinsic frequency. Note that $\delta \Omega(r)=\Omega(r)-\Omega_{t}$ where $\Omega_{t}$ is the rotation rate at the top of the radiative zone. Near a critical layer (i.e. where $\hat{\omega}=0$ ), we will suppose that the considered wave component is totally dissipated and deposits all the angular momentum that it carries into the medium, so that it cannot go deeper in the star.

\section{Efficiency of the transport by IGW}

\subsection{Stellar models and assumptions on the rotation profile}

We consider an evolutionary sequence of $1.3 \mathrm{M}_{\odot}$ stellar models computed with the evolution code CESTAM [4] that covers the subgiant branch and the beginning of the ascent of the red giand branch. Their location in the Hertzsprung-Russel diagram is plotted in figure 1. The chemical composition follows the solar mixture as given in [14], with the initial helium and metal abundances $Y_{0}=$ 0.25 and $Z_{0}=0.013$. We used the NACRE nuclear reaction rates and the OPAL2005 equation of states and opacity tables. The convection was modeled by the mixinglength theory parametrized with $\alpha_{M L T}=1.65$. We did not consider microscopic diffusion, overshooting nor rotation. To compute Eq. (3), we assume that the plume lifetime is 


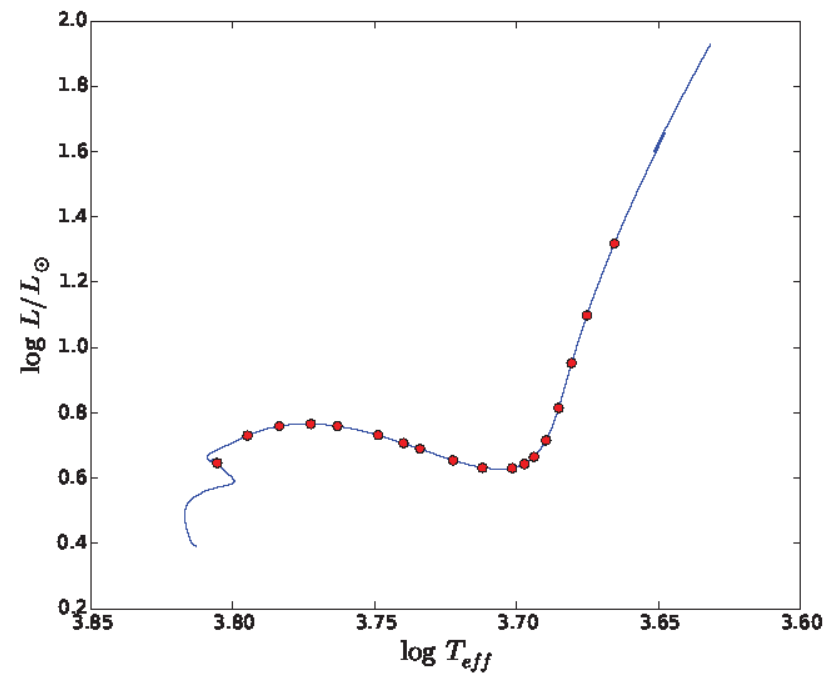

Figure 1: Location in the Hertzsprung-Russel diagram of the $1.3 \mathrm{M}_{\odot}$ stellar models used in this work (red filled circles). The evolutionary track, from the ZAMS to the top of the red giant branch, is represented by the solid blue line.

close to the convective timescale at the base of the convective zone as given by the MLT, i.e. $v_{p} \approx \omega_{M L T} \sim 1 \mu \mathrm{Hz}$ for all the considered models. In addition, we fix the plumes filling factor at a reasonable value $\mathcal{A} \approx 0.1$, as observed in the uppermost layers of numerical simulations of the Sun [e.g. 15]. All the other quantities are directly estimated using the equilibrium internal structure from the stellar models [see 13, for details].

As seen in Eq. (4), the total wave flux depends on the differential rotation in the radiative zone via Eq. (5). As shown by [13] in the solar case, its amplitude can have strong consequences on the transport by IGW. We will then assume a given rotation profile for each stellar model. Doing so, we assume that the rotation rate is low enough to have no effect on the stellar equilibrium structure. Nevertheless, little is known about the shape of the rotation profile in evolved stars. By assuming a priori, first, that a mechanism prevents the core contraction from developing a strong differential rotation in the radiative zone all along the star lifetime (as expected from the observations), and second, that this latter forces a quite smooth profile, it leads us to consider a rotation profile in the form

$$
\delta \Omega(r)=\Delta \Omega \cos ^{2}\left(\frac{\pi}{2} \frac{r}{r_{t}}\right) \quad \text { for } \quad r<r_{t},
$$

with $\Delta \Omega$ the amplitude of the differential rotation between the center and the top of the radiative zone. The arbitrary shape of such a rotation profile is illustrated in figure 2 . We thus assume a decreasing rotation rate from the core to the envelope and the $\cos ^{2}$ function ensures a smooth profile at the center and near the base of the convective zone. Using such a synthetic profile is questionable, but it will give us a first hint about the efficiency of the transport by IGW in subgiant and red giant stars while considering different values for $\Delta \Omega$.

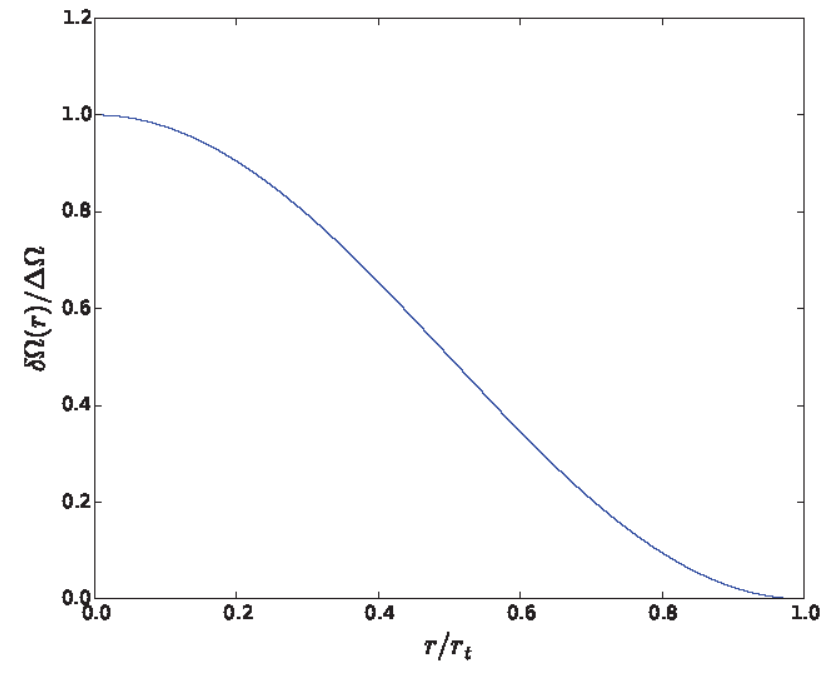

Figure 2: Normalized profile of the internal rotation rate as assumed in this work in the radiative zone of the stars (see Eq. (7)). It is represented as a function of the radius normalized by $r_{t}$, the radius at the top of the radiative zone.

\subsection{Timescale comparison and consequences}

\subsubsection{Stars at the beginning of the ascent of the RGB}

As an example, we consider in the following the case of a model with $\log T_{\text {eff }}=3.68, \log L / L_{\odot}=0.95$ and $\log g=3.28$, which is located at the beginning of the ascent of the red giant branch. In figure 3 (left panel), the characteristic timescale of evolution of the rotation rate due to action of IGW, Eq. (2), is compared to the contraction/dilatation timescale, Eq. (1), as a function of the radius in the radiative zone. The values for $\Delta \Omega$ are chosen between 0 and $12 \mu \mathrm{rad} \mathrm{s}^{-1}$, with the upper limit slightly above the maximum value given by the observations $[2,3]$. As we can see, the wave-driven timescale drastically increases in average as the radius decreases and is still higher than the contraction/dilatation timescale below the H-burning shell regardless the value of $\Delta \Omega$ (in the considered range). Given that the result holds for values of the differential rotation that are consistent with the observations, we can conclude that IGW generated by penetrative convection are likely not to be responsible for the low rotation rates observed in red giant stars. Indeed, as showed in [9] in the case of the IGW generated by turbulent pressure, a strong radiative damping caused by the increase of the Brunt-Väisälä frequency as the stellar core contracts prevents IGW from reaching the innermost layers and modifying the core rotation. As we can note in figure 3 , the wave-driven timescale reaches a local minimum just above the H-burning shell, which indicates a large deposit of angular momentum and a strong damping. Neverthless, we do not exclude that these waves damped near the H-burning shell may interact with the meridional circulation and boost the extraction of angular momentum in the core, but this will have to be tested with a more exhaustive calculation. 

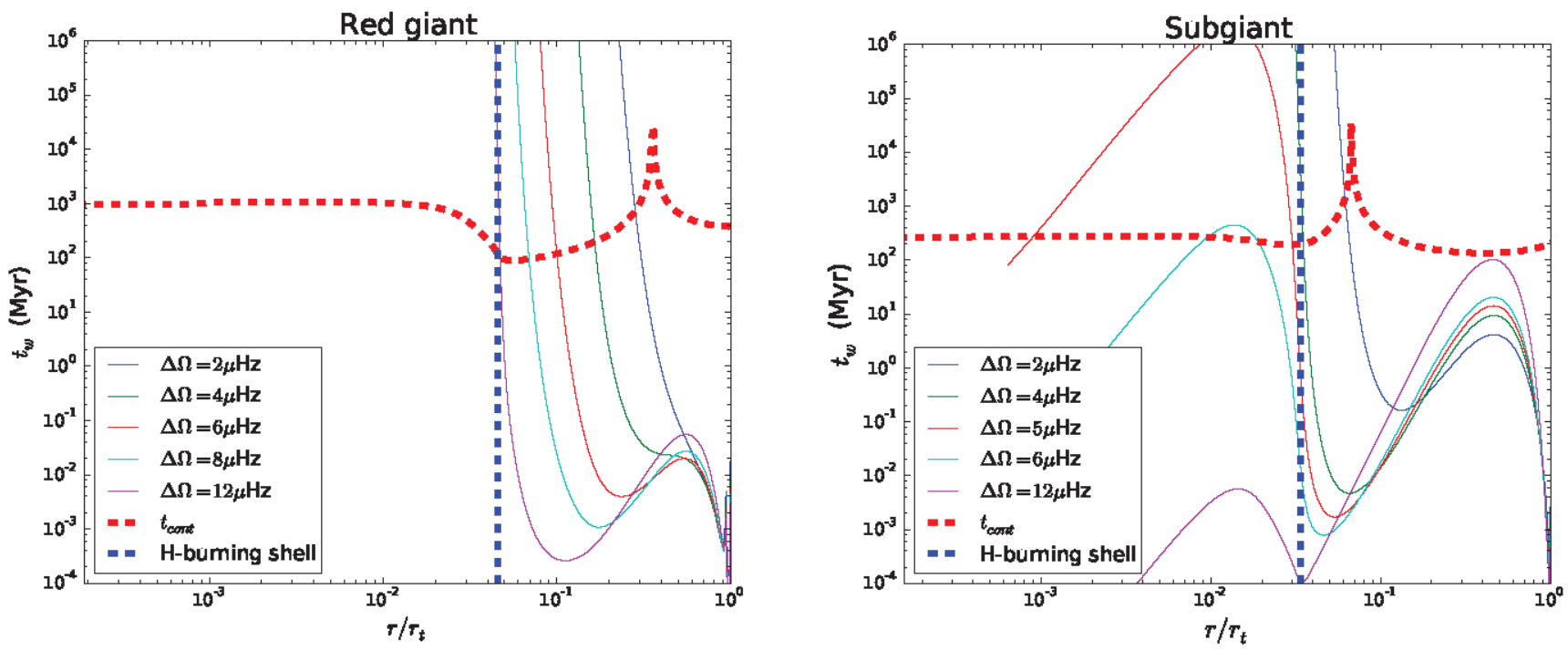

Figure 3: Left: Characteristic wave-driven timescale (solid lines), computed using Eq. (2), as a function of the normalized radius in the radiative zone of a $1.3 \mathrm{M}_{\odot}$ red giant model, with $\log T_{\text {eff }}=3.68$ and $\log L / L_{\odot}=0.95$. Colors correspond to different values for the amplitude of the differential rotation, $\Delta \Omega$. The red and the blue dashed lines represent the contraction/dilatation timescale and the location of the H-burning shell, repectively. Right: Same as the left panel, but for a $1.3 \mathrm{M}_{\odot}$ subgiant model, with $\log T_{\text {eff }}=3.71$ and $\log L / L_{\odot}=0.63$.

\subsubsection{Subgiant stars}

For the subgiant stars, we consider as an illustration the case of a model with $\log T_{\text {eff }}=3.71, \log L / L_{\odot}=0.63$ and $\log g=3.72$. The comparison of both timescales is plotted in figure 3 (right panel) throughout the radiative zone of the star. We can see that the situation is similar as the red giant case for low values of the differential rotation. Nevertheless, as $\Delta \Omega$ increases, $t_{w}$ decreases and

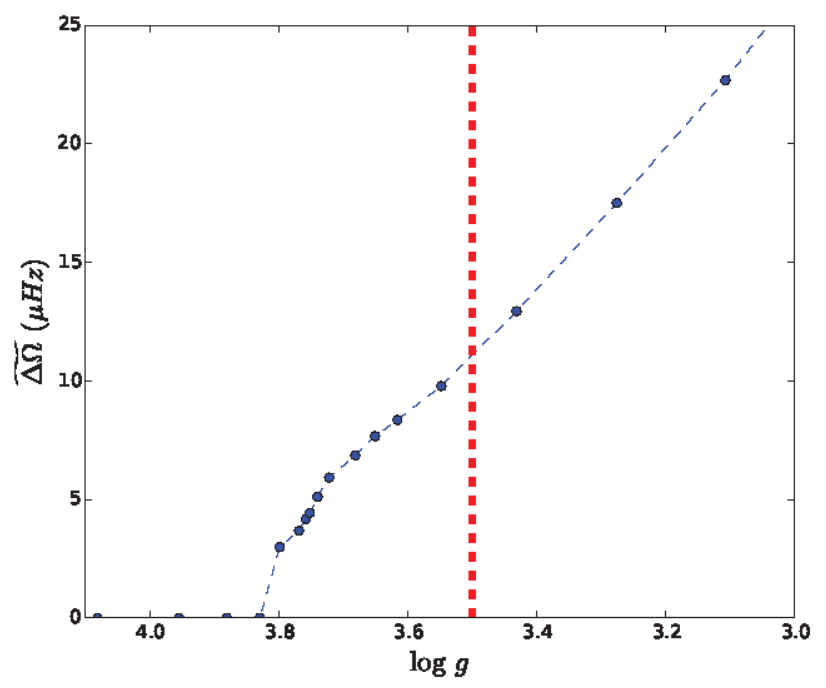

Figure 4: Theoretical threshold for the differential rotation as a function of the $\log \mathrm{g}$. The vertical red dashed line symbolizes the transition between the subgiant phase to the beginning of the RGB. becomes smaller than the contraction timescale all over the region below the H-burning shell as soon as the amplitude of the differential rotation is higher than a threshold, noted $\widetilde{\Delta \Omega}$. In the example plotted in figure 3 (right panel), $\overline{\Delta \Omega} \approx 6 \mu \mathrm{rad} \mathrm{s}{ }^{-1}$. This value is consistent with the mean core rotation rates observed in subgiant stars [2]. For this model, we can then conlude that IGW generated by penetrative convection are likely to play a role in the redistribution of angular momentum and that, at this stage, they have to be taken into account in stellar modeling. In fact, this result can be extended to the whole subgiant branch. In figure 4 , the threshold value $\widetilde{\Delta \Omega}$, above which IGW can counteract the acceleration due to the core contraction in all the region below the H-burning shell, is plotted as a function of the $\log g$. We can see that IGW generated by penetrative convection are quite efficient at the beginning of the subgiant branch, even in the case of very small values for the differential rotation (lower than $0.1 \mu \mathrm{rad} \mathrm{s}^{-1}$ ), because of quite vigourous plume velocities. Further, $\widetilde{\Delta \Omega}$ increases with $\log \mathrm{g}$. Indeed, as the star evolves, the BruntVäisälä frequency increases, leading to an increase in the numerator of the damping depth Eq. (5). Nevertheless, this effect can be balanced by an increase of the amplitude of the differential rotation. Retrograde waves $(\mathrm{m}<0)$ are thus less damped, they can reach the innermost layers to deposit their negative angular momentum and slow down the core rotation. Figure 4 shows that the theoretical values are consistent with the observations in subgiants with values lower than about $10 \mu \mathrm{rad} \mathrm{s}{ }^{-1}$. The values found for stars at the beginning of the red giant branch $\left(\widehat{\Delta \Omega} \approx 17 \mu \mathrm{rad} \mathrm{s}^{-1}\right.$ for the model studied in Sect. 3.2.1) are much higher than the observations [3] and are the consequence of a stronger ra- 
diative damping. As said before, another mechanism must be found for the more evolved red giant stars.

\section{Conclusions}

The comparison of the wave-driven timescale with the contraction/dilatation timescale provides us with a first indication about the efficiency of the transport of angular momentum by IGW generated by penetrative convection throughout the radiative zone of a sequence of $1.3 \mathrm{M}_{\odot}$ models. Plume-induced IGW are shown to be inefficient on their own to slow down the core rotation as soon as the star starts ascending the red giant branch because of a too strong radiative damping near the $\mathrm{H}$-burning shell, in agreement with previous works [e.g. 9]. In contrast, we found that these waves are likely to play a major role on the subgiant branch as soon as the amplitude of the differential rotation is high enough. Indeed, the increase in the Brunt-Väisälä frequency as the star evolves, which should lead to a stronger radiative damping, can be balanced by the increase of the Doppler shift in the retrograde waves frequency. These waves can then deposit their negative angular momentum well below the H-burning shell. This scenario is supported by the strong similarities between the rotation rates observed in low-mass stars and the theoretical thresholds for the differential rotation. These preliminary results need for confirmation from a more thorough study accounting for the uncertainties on the wave energy flux parameters and the assumed shape of the rotation profile. This will be subject to a future paper (Pinçon et al., 2016, in preparation). In a further step, transport by IGW will have to be implemented in a stellar evolution code to model the evolution of the internal rotation along the stars lifetime by taking into account all the transport processes and their interaction with each other.

\section{Acknowledgements}

The authors acknowledge financial support from the EC Project SPACEINN (FP7-SPACE-2012-312844).

\section{References}

[1] S. Deheuvels, R.A. García, W.J. Chaplin, S. Basu, H.M. Antia, T. Appourchaux, O. Benomar, G.R.
Davies, Y. Elsworth, L. Gizon et al., Astrophys. J. 756, 19 (2012), 1206. 3312

[2] S. Deheuvels, G. Doğan, M.J. Goupil, T. Appourchaux, O. Benomar, H. Bruntt, T.L. Campante, L. Casagrande, T. Ceillier, G.R. Davies et al., Astron. \& Astrophys. 564, A27 (2014), 1401.3096

[3] B. Mosser, M.J. Goupil, K. Belkacem, J.P. Marques, P.G. Beck, S. Bloemen, J. De Ridder, C. Barban, S. Deheuvels, Y. Elsworth et al., Astron. \& Astrophys. 548, A10 (2012), 1209. 3336

[4] J.P. Marques, M.J. Goupil, Y. Lebreton, S. Talon, A. Palacios, K. Belkacem, R.M. Ouazzani, B. Mosser, A. Moya, P. Morel et al., Astron. \& Astrophys. 549, A74 (2013), 1211. 1271

[5] T. Ceillier, P. Eggenberger, R.A. García, S. Mathis, Astron. \& Astrophys. 555, A54 (2013), 1305. 5630

[6] P. Kumar, S. Talon, J.P. Zahn, Astrophys. J. 520, 859 (1999), astro-ph/9902309

[7] J.P. Zahn, Rotation and angular momentum transport, in SCORe'96 : Solar Convection and Oscillations and their Relationship, edited by F.P. Pijpers, J. Christensen-Dalsgaard, C.S. Rosenthal (1997), Vol. 225 of Astrophysics and Space Science Library, pp. 187-200

[8] S. Talon, P. Kumar, J.P. Zahn, Astrophys. J. 574, L175 (2002), astro-ph/0206479

[9] J. Fuller, D. Lecoanet, M. Cantiello, B. Brown, Astrophys. J. 796, 17 (2014), 1409.6835

[10] R.B. Stull, Journal of Atmospheric Sciences 33, 1279 (1976)

[11] B. Dintrans, A. Brandenburg, A. Nordlund, R.F. Stein, Astron. \& Astrophys. 438, 365 (2005), astro-ph/0502138

[12] L. Alvan, A.S. Brun, S. Mathis, Astron. \& Astrophys. 565, A42 (2014), 1403.4052

[13] C. Pinçon, K. Belkacem, M.J. Goupil, Astron. \& Astrophys. 588, A122 (2016), 1512.07028

[14] M. Asplund, N. Grevesse, A.J. Sauval, P. Scott, Ann. Rev. Astron. Astrophys. 47, 481 (2009), 0909. 0948

[15] R.F. Stein, A. Nordlund, Astrophys. J. 499, 914 (1998) 